\title{
MPEG Transmission Schemes for a Timed Token Medium Access Control Network
}

\author{
Joseph Kee-Yin Ng \\ Computer Science Department, \\ Hong Kong Baptist University \\ 224, Waterloo Road, Kowloon, Hong Kong. \\ Email: jng@comp.hkbu.edu.hk
}

\begin{abstract}
This paper presents three transmission schemes to improve the transmission of MPEG video over a timed token medium access control (MAC) network. Multiple classes of MPEG video are used in the study. These data are captured from real video programmes and we categorized these video clips according to their traffic burstiness and workload characteristics. The performance measure of the timed token MAC network is in terms of the maximum number of MPEG video streams being transmitted without any frame missing its deadline. This simulation study observed that by employing the Regulated Scheme, we could improve the performance by 28 to 40\%. When applying the Grouping Scheme for the MPEG transmission, we can improve the performance between 142 and 149\%. When we combined the two schemes together, the Regulated Grouping Scheme can further improve the performance up to 153\% which is a dramatic improvement over the original transmission scheme.
\end{abstract}

\section{$1 \quad$ Introduction}

Because of the demands of the videos on network bandwidth, video data needs to be compressed before being transmitted over a computer network. Many compression schemes have been proposed or developed for these variable bit rate (VBR) video services. Of all these video compression schemes, the scheme developed by the Moving Pictures Experts Group (MPEG) is the most notable. By MPEG standard, video information is compressed frame by frame, and the resultant frames should be transmitted and processed under stringent timing constraints. To support such applications on top of a computer network, the underlying transmission scheme must ensure that most, if not all, frames should reach their destinations before their deadlines. Frames arriving late and missing their deadlines will result in a poorer quality of the picture or jumpy video. Hence, it is a tradeoff between video quality and on-time delivery of video frames.

In order to address the issue of on-time video frame delivery, transmission delays for the underlying network must be deterministic and bounded. Here, we adopted the time token medium access control protocol because of its important property of bounded transmission delay. This bounded delay property is a necessity for real-time communications, multimedia computing systems, and industrial process controls. This is why the timed token MAC protocol has been well received and being adopted in several high-bandwidth network standards over the years. These include the Fibre Distributed Data Interface (FDDI) [ANS87a, ANS87b], IEEE 802.4 (Token Bus) [ANS85], the High-Speed Data Bus and the High-Speed Ring Bus (HSDB/HSRB) [COH89, SAE88, UHL91], the Survivable Adaptable Fiber Optic Embedded Network (SAFENET) [GRE89, KOC91, PAI90], and Profibus, a German standard for fieldbus that is widely used in time-critical industrial applications [MIN96]. Besides, many embedded real-time applications also used the timed token MAC protocol as their backbone network.

Furthermore, some of our previous studies [NG91, NG93A, NG93B, NG93C] have shown that the timed token MAC protocol is suitable for real-time communication and multimedia application within 
a local area network (LAN). The main idea behind the timed token MAC protocol is to control the sharing of network bandwidth among the stations for transmitting synchronous messages. That is the bandwidth allocation scheme for synchronous traffic. Among the studies on the bandwidth allocation schemes in a timed token MAC protocol, Agrawal, Chen, Malcolm, and Zhao [AGR94, CHE92, MAL93, ZHA94] proposed a normalized proportional allocation scheme which will give the highest worst case achievable utilization and the utilization bound is at $33 \%$. That is, as long as the total synchronous traffic is no more than $33 \%$, these synchronous messages are guaranteed to be transmitted before their deadlines. Since this bandwidth allocation scheme is well accepted in the real-time communication community, we adopted this scheme for our MPEG transmission experiments.

On the other hand, the variable bit rate (VBR) video encoding schemes as well as the MPEG-I video transmission over a computer network have been studied extensively. Ott et al.[OTT92] and Lam et al.[LAM94] proposed smoothing schemes for VBR video. Reibman and Berger [REI92] and Reininger et al.[REI93] studied the problem of transporting/multiplexing VBR/MPEG video over ATM networks. Pancha and Zarki[PAN92, PAN93] studied the MPEG-I video coding standard for transmission of VBR video and the performance of variable bandwidth allocation schemes for VBR MPEG-I video. Furthermore, there were also extensive studies on MPEG video characterization and modeling by Ismail, Lambadaris, and Devetsikiotis, Izquierdo and Reeves, and Krunz, Sass, and Hughes [ISM95, IZQ95, KRU95].

In this paper, we constructed a series of simulation experiments to study the performance of a timed token MAC network in supporting MPEG-I video transmissions. This paper proposed three schemes -- the Regulated Scheme, the Grouping Scheme and the Regulated Grouping Scheme -- for improving the real-time support of MPEG-I transmission on a high-speed network with a timed token MAC protocol. What makes these experiments different from the others is that the video data we used are captured from various TV programmes. We categorized these video clips according to their workload characteristics -- average frame size, maximum frame size, and I:P:B ratio -- and studied their effect on the transmission schemes and the underlying network.

Reader should notice that by MPEG standard, we are referring to the MPEG-I standard as specified by ISO/IEC-11172. From here and onward, we will omit the suffix and used MPEG instead of MPEG-I. However, reader should note that our proposed transmission schemes are also applicable to MPEG-II, and other VBR video streams. In fact, these transmission schemes can be generalized and applicable to other network protocols for real-time communication applications and time critical industrial controls.

The remainder of this paper is organized as follows: Section 2 describes the system model and the timed token MAC protocol. Section 3 describes the MPEG video streams, encoding parameters, and their classification. Section 4 discusses the motivation, observations, and describes the proposed transmission schemes. Section 5 describes the simulation experiments, performance metrics, and presents the simulation results. Finally, Section 6 provides a summary of this study and discusses our future research direction.

\section{System Model \& The Timed Token MAC Protocol \\ 2.1 System Model}

We consider a network with $n$ stations connected by point-to-point links forming a ring. The medium access control is controlled by token-polling from station to station. In a network with a timed token MAC protocol, messages are classified into two categories: synchronous and asynchronous messages. Synchronous messages arrive or ready to be transmitted at regular intervals (periodic) and are 
associated with deadline constraints. On the other hand, asynchronous messages are non-periodic and do not have a real-time requirement. The main idea of the timed token MAC protocol was presented by Grow [GRO82] and further studied by Ulm [ULM82].

It was shown in [AGR94] that any arbitrary ring network where a node may have zero, one or more streams of synchronous message could be transformed into an equivalent network with one stream per node. Therefore, without loss of generality, we assume that there is one synchronous message stream at each node but a station can have zero, one or more nodes for transmitting synchronous message streams.

The followings are some notation that will be used throughout this paper. We consider a system having a synchronous message set, $M$, consists of $m$ synchronous message streams: $S_{1}, S_{2}, \ldots, S_{m}$. That is

$$
M=\left\{S_{1}, S_{2}, \ldots, S_{m}\right\}
$$

We denote $P_{i}$ to be the period of the synchronous stream $S_{i}, i=1,2, \ldots, \mathrm{m}$, and the deadline for the message in the stream $S_{i}$ be at the end of the period in which it arrives. Thus, if the message arrives at time $t$, its deadline will be at $t+P_{i}$.

\subsection{The Timed Token MAC Protocol}

The prime objective of the timed token MAC protocol is to control the token rotation time. The value of the Target Token Rotation Time (TTRT) has to be determined when the network is initialized. This TTRT gives the expected value of the token rotation time for the protocol. In 1987, Sevik and Johnson [SEV87] had shown that the worst case time interval between two consecutive token visits at a node in a timed token MAC protocol can be as much as 2 TTRT. Therefore, we have to set the TTRT for the synchronous message stream $S_{i}$ as

$$
T T R T \leq \frac{P_{i}}{2} \quad \text { for } 1 \leq i \leq m
$$

where $P_{i}$ is the period of $S_{i}$ and $S_{i} \in M$.

Another protocol parameter is the maximum time a station is allowed to transmit the synchronous message when a token is received. We denote $H^{i}$ as the synchronous bandwidth assigned to station $i$. Recall that every station can have zero, one ore more synchronous streams to transmit. Thus, each station can have a different value of $H$ depending on the number of synchronous streams and the workload for each stream in the station. Furthermore, the synchronous bandwidth, $H^{i}$, is subjected to the following protocol constraint

$$
\sum_{i=1}^{n} H^{i} \leq T T R T-\tau
$$

where $\tau$ is the time a token takes to cycle through the network when all $n$ stations have nothing to send.

Besides the two parameters, TTRT and $\mathrm{H}$, each station on the network has two countdown timers -- a token rotation timer (TRT) and a token holding timer (THT), and a late counter (LC). At network initialization, THT and LC are set to zero. TRT is set to the value of TTRT and starts counting. The TRT counter always counts down until TRT $=0$. When TRT is zero, the late counter (LC) is incremented and TRT starts counting down from TTRT again. 
At a station, a token is considered to arrive early if $\mathrm{LC}=0$ upon its arrival and is considered to be late if LC>0. When a token arrives early at a station, the THT is set to the remaining time on the TRT counter, LC is reset, and TRT is reset to the value of TTRT and counts down again. This station is allowed to transmit synchronous messages for a maximum of $\mathrm{H}$ units of time. After transmitting the synchronous messages (if any), THT starts counting down. As long as THT $>0$ and TRT $>0$, this station is allowed to transmit asynchronous messages. After the asynchronous transmissions (if any), the station passes the token to the next station downstream. On the other hand, if the token arrives late, LC will be reset to zero, and TRT will continue to count down without resetting. No asynchronous message is sent, but the station is allowed to send synchronous messages for a maximum time of $\mathrm{H}$. After the transmission, the token will be released to the next station. [NG91, NG93A, NG93B, NG93C]

In this paper, we set the two parameters TTRT and $H_{j}$ for each video stream $S_{j}$ where $j=1,2, \ldots$, $\mathrm{m}$, according to the normalized proportional allocation scheme proposed by Wei Zhao et al [AGR94, CHE92, MAL93, ZHA94]. Their work claimed that with the normalized-proportional allocation scheme for the timed token MAC protocol, one can achieve the highest worst-case achievable utilization of $33 \%$.

Since we are dealing with video streams encoded at a period of $P$ (i.e. $30 \mathrm{fps}$ ), each synchronous message stream $S_{j}$ in our system has $P_{j}=P=1 / 30$ second. Thus, we set the TTRT as follows

$$
\text { TTRT }=\frac{P}{2}
$$

Because the MPEG video is a variable bit rate message stream, the transmission time for each frame within the period varies. Assume a video stream $S_{j}$ of $x$ frames in length, we denote the transmission time needed for each frame in $S_{j}$ as $C_{j, i}$ where $i=1,2, \ldots, \mathrm{x}$. We also denote $C_{j}^{\max }$ as the maximum transmission time required to transmit a frame in video stream $S_{j}$. Then we have

$$
C_{j}^{\max }=\max _{i=1}^{x}\left\{C_{j, i}\right\}
$$

According to the normalized proportional allocation scheme, the synchronous bandwidth, $H_{j}$, assigned to each stream $S_{j}$ follows the formula

$$
H_{j}=\frac{C_{j}^{\max } / P}{U} \times(\text { TTRT }-\tau)
$$

where $C_{j}^{\max }$ is the maximum transmission time required to transmit a frame in video stream $S_{j}$; and

$$
U=\sum_{j=1}^{m} \frac{C_{j}^{\max }}{P}
$$

which is the total utilization of all video streams in the system $\left\{S_{1}, S_{2}, \ldots, S_{m}\right\}$

Substitute (2.7) into (2.6), we have

$$
H_{j}=\frac{(T T R T-\tau)}{m}
$$

where $m$ is the number of video stream in the system. 


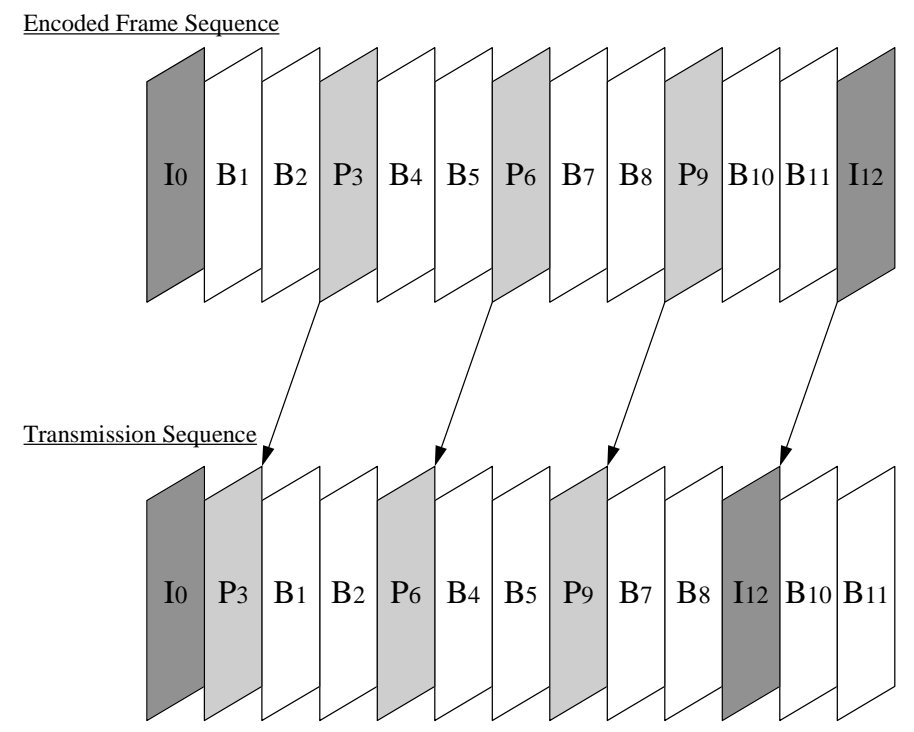

Figure 1. The encoded frame sequence versus the transmission sequence of an MPEG stream.

The reason why the normalized proportional allocation scheme is reduced to a equal partition allocation scheme is because all video streams in the system are having the same period, $P$, of $30 \mathrm{fps}$.

With the bandwidth allocation scheme above, we can set the maximum time allowed to send synchronous messages for station $i$ to be,

$$
H^{i}=\sum H_{k}
$$

for all $k$ that are synchronous messages in station $i$.

\section{MPEG Video Stream}

MPEG was originally designed for storing video and audio on digital media, however, MPEG compression is also suitable for transmitting video frames over a computer network. The basic idea of this compression scheme is to predict motion from frame to frame in the temporal direction, and then to use discrete cosine transforms (DCT) to organize the redundancy in the spatial directions. In MPEG standard, there are three types of frames. The "I" frames or intra-frames are simply frames coded as still images, not using any past history. Then there are "P" frames or predicted-frame that are predicted from the most recently reconstructed I- or P-frame. Each macro-block in a P-frame can either come with a vector and difference DCT coefficients for a close match in the last I- or P-, or it can just be "intra" coded if there was no good match. Lastly, we have the " $\mathrm{B}$ " frames or bi-directional-frames. These are predicted from the nearest two I- or P-frames, one in the past and one in the future. In normal cases, the frame size of an I-frame is larger than that of a P-frame; and the frame size of a P-frame is larger than that of a B-frame. The encoded frame sequence usually follows the pattern of "IBBPBBPBBPBB" and repeats itself.

However, since the B-frame needs a subsequent P-frame or I-frame to reconstruct the current frame, for the decoder to work, one have to send the first P-frame before the first two B-frames. Figure 1 shows the relationship between the encoded frame sequence and the transmission sequence for a typical MPEG video stream. Hence, except for the very first I-frame, the transmission sequence follows the pattern of "PBBPBBPBBIBB" and repeats itself. Therefore, in our simulation experiment, we intended to ignore the first I-frame and start our simulation from the second transmitted frame. 


\begin{tabular}{|l|l|}
\hline \multicolumn{2}{|c|}{ MPEG-I Parameters } \\
\hline Dimension & 320 X 240 \\
\hline Frame Rate & 30 Frames / Second \\
\hline Constant Frame Type Sequence Pattern & IBBPBBPBBPBB \\
\hline Factor of Quantization [scales quantization matrix] & 5.33 \\
\hline Number of Macro-blocks [width X height = sum] & 20 X 15=300 per Frame \\
\hline I-Frame Q Factor & 4 \\
\hline P-Frame Q Factor & 4 \\
\hline B-Frame Q Factor & 8 \\
\hline Cost function to be minimized by block-matching & $\begin{array}{l}\text { Sum of difference magnitudes } \\
\text { (Manhattan distance) }\end{array}$ \\
\hline Block matching & Logarithmic block matching \\
\hline
\end{tabular}

Table 1. A table summarizing the parameters used to generate the MPEG video clips.

In our experiments, we have a total of 52 video clips captured from different $\mathrm{TV}$ programmes with different content. Each clip is captured in 24-bit true color, with a resolution of $320 \times 240$ at 30 frames per second. The resultant frame sequence was then compressed according to the MPEG standard. The parameters used for converting the video sequence to MPEG format is summarized in Table 1 above. According to the workload characteristics and the video content, we categorized these MPEG video clips into different categories and types as shown in Table $2 \& 3$, respectively.

\begin{tabular}{|l|c|c|c|l|}
\cline { 2 - 3 } \multicolumn{1}{l|}{} & \multicolumn{2}{c|}{ Frame Size (bits) } & \multicolumn{2}{l|}{} \\
\hline Category & Maximum & Average & Comp Ratio & Remarks \\
\hline News Report & 83,520 & 23,279 & $1.27 \%$ & Mainly mouth movements \\
\hline Test Card & 122,548 & 26,568 & $1.44 \%$ & Still images \\
\hline $\begin{array}{l}\text { Computer } \\
\text { Animation }\end{array}$ & 120,027 & 28,296 & $1.54 \%$ & Simple movement \\
\hline Music Video & 125,396 & 29,124 & $1.58 \%$ & Slow songs music video \\
\hline Soap Opera & 132,602 & 31,391 & $1.70 \%$ & $\begin{array}{l}\text { Camera flipping back and forth } \\
\text { during conversation }\end{array}$ \\
\hline Ball Games & 140,154 & 38,546 & $2.09 \%$ & $\begin{array}{l}\text { Tennis, football, soccer, baseball, } \\
\text { and badminton }\end{array}$ \\
\hline TV Commercials & 152,579 & 38,522 & $2.09 \%$ & $\begin{array}{l}\text { Dynamic changes of screens \& } \\
\text { backgrounds }\end{array}$ \\
\hline Car Racing & 131,452 & 42,160 & $2.29 \%$ & Chase camera, fast movements \\
\hline
\end{tabular}

Table 2. A table summarizing the MPEG video clips used in our simulation experiments.

\begin{tabular}{|c|l|c|c|c|c|c|}
\hline Type & Content & I:P:B Ratio & Ave. Frame Size (bit) & Ave-I & Ave-P & Ave-B \\
\hline I & $\begin{array}{l}\text { Auto racing, TV } \\
\text { Commercials }\end{array}$ & $4: 3: 1$ & 40507 & 85830 & 75219 & 21590 \\
\hline II & $\begin{array}{l}\text { Various kinds of } \\
\text { ball games }\end{array}$ & $6: 5: 1$ & 38546 & 103593 & 74844 & 16519 \\
\hline III & $\begin{array}{l}\text { Animation, MTV, } \\
\text { Soap opera }\end{array}$ & $9: 5: 1$ & 29960 & 92523 & 59483 & 10826 \\
\hline IV & $\begin{array}{l}\text { News, Testcard, } \\
\text { Tele-market }\end{array}$ & $12: 6: 1$ & 24594 & 94301 & 44809 & 8072 \\
\cline { 2 - 6 } & Overall & $6: 4: 1$ & 34978 & 95536 & 67309 & 15028 \\
\hline
\end{tabular}

Table 3. Categorize the MPEG video into four types according to their I:P:B ratios. 


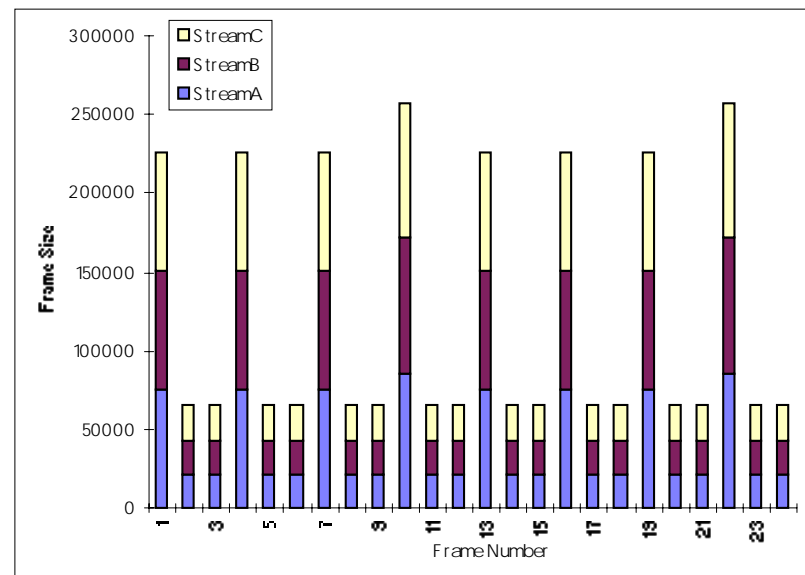

Figure 2. Bandwidth utilization for Type-I MPEG stream without Regulated Delay.

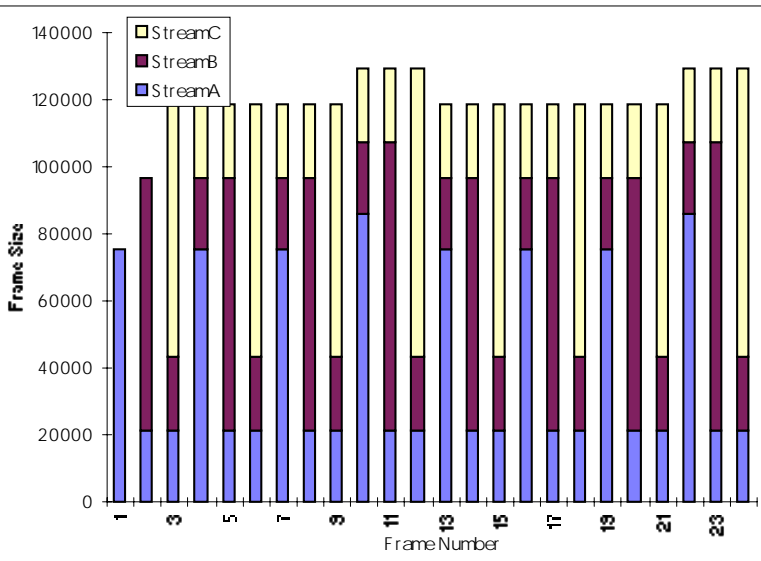

Figure 3. Bandwidth utilization for Type-I MPEG stream with Regulated Delay.

\section{4}

\section{The MPEG Transmission Schemes}

In this section, we present three transmission schemes in order to improve the transmission of MPEG video streams over a timed token MAC network. As we are dealing with stored video stream instead of real-time encoding stream like in teleconferencing, the video frames are always ready for transmission. With the default transmission scheme, traffic is non-regulated so that stations could be completing for the network bandwidth at the same time. The performance of this "best effort" transmission scheme is worsen by the MPEG video's variable bit rate nature. By these observations, we are motivated to propose new transmission schemes so as to regulate the network traffic at the sending station and thus, better utilized the network bandwidth. The approach for each of the scheme is described below.

\subsection{The Regulated Scheme}

As mentioned in Section 3, when ignoring the very first I-frame, the transmission sequence of a MPEG file follows the pattern of "PBBPBBPBBIBB". Because of the differences in size for the I-, P-, and B-frames, the demand of bandwidth grows and shrinks following the same pattern of the transmission sequence. Hence, in the worst case, if every MPEG stream happens to start transmitting at the same time, the demand of bandwidth for transmitting the first wave of P-frame is much greater than transmitting the second frame, a B-frame, 1/30 second later. The situation is even worse when there comes a time such that every station needs to transfer an I-frame (the 10-th frame) at the same time.

Figure 2 shows the demand of bandwidth when transmitting three Type-I videos at the same time. Within the first frame-time, three P-frames $(3$ X $75219 / 1024=220 \mathrm{Kbit})$ have to be transmitted. The demand of bandwidth then shrinks to about 3 X $21590 / 1024=63$ Kbit for the next frame-time and the one after it, then the demand of bandwidth grows again because of the transmission of the P-frames again. Since the network have to guarantee each frame meeting its deadline, each station should be allocated a bandwidth equals to its maximum demand. Hence, a lot of the available bandwidth is wasted.

In order to make good use of the available bandwidth, we examined 3 Type-I MPEG streams as shown in Figure 2. The problem is due to transmitting MPEG streams at the same time and that the transmission sequence follows a specific pattern. If we intentionally delay (or regulate) the transmission of the second MPEG stream (StreamB) by one frame-time, and the third MPEG stream (StreamC) by two frame-times, we can better utilize the bandwidth as shown in Figure 3. One should notice that delaying the start of a transmission by one or two frame-time is hardy recognizable by ordinary users. From Figure 2 , in order to support the 3 Type-I MPEG streams, a maximum demand of 3 X $85830 / 1024$ X $30=7.37$ 
Mbps is needed and only $47 \%$ of the bandwidth is utilized. By applying our Regulated Scheme, from Figure 3, an estimated maximum demand of $(85830+2$ X 21590) / 1024 X $30=3.69 \mathrm{Mbps}$ is needed and $94 \%$ of the bandwidth can be utilized.

Thus, we propose the Regulated Scheme as follows:

Consider a discrete-time model in frame-time, for a given MPEG video stream, $t \in\{1,2, \ldots, x\}$ where $x$ is the total number of frames in the video stream, we define a transmission function $F(t)$ such that:

$$
F(t)=\left\{\begin{array}{cc}
C_{t} & t \in\{1,2, \ldots, x\} \\
0 & \text { otherwise }
\end{array}\right.
$$

where $C_{t}$ is the size of the t-th frame.

We also define another transmission function $F_{k}(t)$ such that

$$
F_{k}(t)=F(t-k)
$$

If we transmit 3 MPEG streams in the system at the same time and let $Q^{3}(t)$ be the transmission function for this 3-MPEG streams system. We have

$$
Q^{3}(t)=3 \times F(t)
$$

If we let $R^{3}$ to be the peak rate of this 3-MPEG streams system, we have

$$
R^{3}=3 \times \max \{F(t)\}=3 \times C_{\max }
$$

where $C_{\max }$ is the maximum size of a frame within the video stream.

If we intentionally delay the transmission of the second MPEG stream by one frame-time, and the third MEPG stream by two frame-times, we have

$$
Q^{3}(t)=F(t)+F_{1}(t)+F_{2}(t)
$$

and the peak rate for the Regulated Scheme for this 3-MPEG streams system is:

$$
R_{R S}^{3}=3 \times \max \left\{F(t)+F_{1}(t)+F_{2}(t)\right\}
$$

which is the maximum size of a IBB or PBB group of frames in the video stream. have

With (4.4) and (4.6), and the fact that I-frame > P-frame > B-frame in term of frame size, we

$$
3 \times \max \{F(t)\}>\max \left\{F(t)+F_{1}(t)+F_{2}(t)\right\}
$$

Thus, the burstiness of the video stream is smoothed and the bandwidth is better utilized.

Furthermore, since the Group of Pictures (GOP) of an MPEG stream follows the pattern of "PBBPBBPBBIBB", we can extend our Regulated Scheme up to 11 frame-time delay. Then we have,

$$
R_{R S}^{12}=\max \left\{F(t)+F_{1}(t)+F_{2}(t)+\ldots+F_{11}(t)\right\}
$$

which is the maximum size of a GOP in the video stream.

For this 12-MPEG streams system, the peak rate for the default transmission scheme is:

$$
R^{12}=12 \times \max \{F(t)\}
$$


From (4.8) and (4.9), we know that $R^{12}>R_{R S}^{12}$, hence, the bandwidth could be further utilized.

Practically, we can only apply the Regulated Scheme locally within a single station. However, we can also investigate whether the scheme will perform better if we apply it globally. Hence, in our simulation experiment, besides the local Regulated Scheme, we also have a global Regulated Scheme that assumes we have such global information at the transmission time.

\subsection{The Grouping Scheme}

According to our MPEG analysis, a typical MPEG stream is a variable bit rate stream and in general, has a I:P:B ratio of 6:4:1. This fluctuation on bandwidth demand is not good for the timed token MAC network. Therefore, in order to provide a better real-time transmission support, the main objective of this scheme is to smooth out the variable bit rate transmission. This can be achieved by grouping subsequent frames together and form a mega-frame. By looking at the transmission sequence and their average frame size, combining IBB and PBB together should smooth out the transmission of a video stream.

For the Grouping Scheme, we group three frames (IBB or PBB) together and send them as a mega-frame with a period of 3 frame-time. Here we define a transmission function $G(t)$ for a video stream as:

$$
G(t)=\left\{\begin{array}{cc}
g_{t} & t \in\{1,2, \ldots, x\} \\
0 & \text { otherwise }
\end{array}\right.
$$

where $g_{t}$ is the average frame size of a IBB or PBB frame-group. That is

$$
g_{t}=\{F([t / 3])+F([t / 3]+1)+F([t / 3]+2)\} / 3
$$

For the Grouping Scheme, the peak rate of the resultant stream is,

$$
R_{G S}=\max \{I B B, P B B\} / 3=\max \left\{F(t)+F_{1}(t)+F_{2}(t)\right\} / 3
$$

which is smaller than the peak rate of the original stream $(R=\max \{F(t)\})$.

If we group 6 frames and 12 frames together, the peak rate of the two resultant video stream would be $\max \left\{F(t)+F_{1}(t)+\ldots+F_{5}(t)\right\} / 6$, and $\max \left\{F(t)+F_{1}(t)+\ldots+F_{11}(t)\right\} / 12$, respectively.

\subsection{The Regulated Grouping Scheme}

We intended to combine the two schemes together and investigate if a combined scheme can further improve the performance. Like the Grouping Scheme, we group 3 frames together and form a mega-frame. Thus, we end up with 4 such mega-frames per GOP. We then apply the Regulated Scheme to this mega-frame stream. When we are transmitting 4 mega-frame streams at the same time, the first stream will be transmitted as usual. The other 3 streams will be intentionally delay by 1,2, and 3 megaframe time (3 frame-time in this case) from their starts.

The transmission function for the first mega-frame stream is the same as the one for the Grouping Scheme, that is equation (4.10). However, the transmission function for the second, third, and forth mega-frame streams will be defined as:

$$
G_{k}=G(t-3 k)
$$

where $k=1,2,3$. 


\begin{tabular}{|l|l|}
\hline \multicolumn{2}{|c|}{ Network Parameters } \\
\hline Propagation speed & $3 \times 10^{8} \mathrm{~m} / \mathrm{s}$ \\
\hline Network length & $1000 \mathrm{~m}$ \\
\hline Packet size & $2048 \mathrm{bit}$ \\
\hline Number of workstations & 20 \\
\hline Distribution of MPEG video streams & Evenly distributed \\
\hline Data transfer rate & $100 \mathrm{Mbps}$ \\
\hline Token length & 24 bit \\
\hline Protocol overhead & 160 bit \\
\hline Network preamble & 16 bit \\
\hline
\end{tabular}

Table 4. Network protocol parameters for all our simulations.

With this Regulated Grouping Scheme, the peak rate of the 4 mega-frame stream system is:

$$
R_{R G S}^{4}=\max \left\{G(t)+G_{1}(t)+G_{2}(t)+G_{3}(t)\right\}=\max \{G O P\} / 3
$$

Compare (4.14) with the peak rate of a regular 4-stream system in which $R^{4}=4 \times \max \{F(t)\}$, we have $R^{4}>R_{R G S}^{4}$, under the general assumption that, an I-frame is bigger than a $\mathrm{P}$-frame and a $\mathrm{P}$ frame is bigger than a B-frame in term of frame size.

Similarly, we can also group 6 frames at a time and form 2 mega-frames per GOP and intentionally delay the second stream by 1 mega-frame time. Furthermore, this modified Regulated Scheme can also be applied either locally, or globally. Therefore, in our simulation experiments, we simulated both a local and a global Regulated Grouping Scheme for the MPEG video transmission.

\section{$5 \quad$ Simulation Experiment \& Results}

Because of the variable bit rate (VBR) in a MPEG video stream, it is difficult to tell which transmission scheme works the best by looking at the peak rate equation for each scheme. So far the peak rate equations only show that the proposed schemes are better than the default transmission scheme but cannot identify which one of the three will give the best performance. Therefore, we have constructed a series of experiments to find out the performance improvement among the three proposed schemes as compared with the default "non-regulating" transmission scheme.

\subsection{Simulation Setup \& Performance Metrics}

We use a discrete event simulation model to simulate a timed token MAC network with 20 stations. The simulation program is written in $\mathrm{C}++$ and run in a SGI/IRIX environment. Table 4 lists the fixed values of the network parameters we have chosen to use in our simulations. Specific values are chosen either because the values are given by the specification of the timed token MAC protocol or because the actual value of the parameter has little effect on the simulation results. For example, we ran our experiments with different packet sizes ranging from 2048 bit to 16Kbit and found out that the packet size has little effect on the performance. Therefore, from this point onward, we discuss the results based on a timed token MAC network with a packet size of 2048 bit.

To be more specific on the network simulation, each simulation run refers to a particular video stream. We have a total of 52 video clips, thus, we ran 52 simulations for each transmission scheme. For each video stream and for a particular transmission scheme, we estimate the maximum number of streams the network can possibly support according to the video stream's average workload. Then by a binary 
search, we can find out how many streams a particular transmission scheme can support without any of the video frame missing its deadline.

As mentioned above, for the performance measure, we use the maximum number of MPEG video streams a timed token MAC network can support under a particular transmission scheme. Supporting an MPEG video stream means that the network can deliver the frames without any of them missing its deadline.

Besides the maximum number of MPEG stream can be supported, we also look at the performance improvement $(P I)$ for each transmission scheme with respect to the default transmission scheme. Performance improvement $(P I)$ is defined as:

$$
P I=\frac{\left(N_{\text {scheme }}-N_{\text {default }}\right)}{N_{\text {default }}} \times 100 \%
$$

where $N_{\text {scheme }}$ is the maximum number of streams supported by one of the proposed transmission scheme and $N_{\text {default }}$ is the maximum number of streams supported by the default transmission scheme.

\subsection{Simulation Results}

All the simulation results are listed in Appendix A. Knowing the maximum number of MPEG streams can be supported by a particular transmission, the performance improvement (PI) for each of the 52 video streams were calculated.

\begin{tabular}{|l|c|c|c|c|}
\cline { 2 - 5 } \multicolumn{1}{c|}{} & \multicolumn{2}{c|}{ Local } & \multicolumn{2}{c|}{ Global } \\
\hline Type & Max. 2 Frame & Max. 11 Frame & Max. 2 Frame & Max. 11 Frame \\
\hline I & $35.28 \%$ & $22.64 \%$ & $46.80 \%$ & $36.65 \%$ \\
\hline II & $21.19 \%$ & $22.61 \%$ & $32.83 \%$ & $32.97 \%$ \\
\hline III & $44.99 \%$ & $30.99 \%$ & $43.33 \%$ & $40.31 \%$ \\
\hline IV & $58.88 \%$ & $60.39 \%$ & $51.75 \%$ & $42.79 \%$ \\
\hline Overall & $35.12 \%$ & $28.38 \%$ & $40.84 \%$ & $36.95 \%$ \\
\hline
\end{tabular}

Table 5. Performance improvement by the Regulated Schemes.

\subsubsection{The Regulated Scheme}

In order to verify whether the Regulated Scheme works out as planned, we ran a series of simulation and summarized the results in Table 5. Table 5 shows the performance improvement when we applied the Regulated Scheme to different types of MPEG traffic.

With a maximum delay of 2 frame-time, the overall improvement is $35.12 \%$. If we applied the scheme globally, we can further improve the performance to $40.84 \%$. There is a general trend across the different types of MPEG videos. As the I:P:B ratio changes from 4:3:1 to 12:6:1, the amount of improvement increases. It is not a surprise to see a better performance improvement in Type-IV traffic than the others. The main reason is the video's burstiness as indicates by its I:P:B ratio of 12:6:1 and of its relatively small frame size. The result indicates that the Regulated Scheme performs better on bursty traffic. Table 5 also indicates that the improvement for the scheme with a maximum delay of 11 frametime is not as good as the 2 frame-time delay scheme. 


\begin{tabular}{|l|c|c|c|}
\cline { 2 - 4 } \multicolumn{1}{c|}{} & 3-Frame grouping & 6-Frame grouping & 12-Frame grouping \\
\hline Type & IBB or PBB & IBBPBB or PBBPBB & IBBPBBPBBPBB \\
\hline I & $113 \%$ & $117 \%$ & $120 \%$ \\
\hline II & $136 \%$ & $138 \%$ & $139 \%$ \\
\hline III & $155 \%$ & $161 \%$ & $164 \%$ \\
\hline IV & $191 \%$ & $201 \%$ & $208 \%$ \\
\hline Overall & $142 \%$ & $147 \%$ & $149 \%$ \\
\hline
\end{tabular}

Table 6. Performance improvement by the Grouping Schemes.

\subsubsection{The Grouping Scheme}

Table 6 shows the simulation results of our experiments in terms of performance improvement with the Grouping Schemes. From Table 6, we can see that by grouping IBB and PBB as a mega-frame, Type-I, II, III, IV have an average increase in performance of $113 \%, 136 \%, 155 \%$, and $191 \%$, respectively. One drawn back of this scheme is that when we are sending the mega-frame at a slower rate -- a period of 3-frame time (or 10 frames per second), the mega-frame are guaranteed to meet their deadlines, but the frames within the mega-frame may occasionally miss their internal deadlines. We can easily introduce sub-deadlines checking during a mega-frame transmission but these additional checks might not be necessary in practice. It is because the human eye can hardy recognize a frame which is out of synchronization within 3 frame-time -- that is $1 / 10$ second. Studies by Shah et al [SHA92] have shown that our vision system will recognize a sequence of frames as distinct pictures if the display rate is at 8 frames per second or lower. Between 10 to 12 frames per second, the frame sequence would appear to be choppy. The frame sequence would appear to be continuos when the display rate is over 15 frames per second. Hence, our Grouping Schemes should work out nicely for improving MPEG transmission over a timed token MAC network at the expense of some frames slightly missing their deadlines.

Furthermore, grouping more frames together like the 6-Frame grouping of IBBPBB or PBBPBB and the 12-Frame-grouping of IBBPBBPBBPBB can further improve the performance, but the improvement is not as dramatic as the 3-Frame grouping scheme. One can observed from Table 6 that by the 3-Frame grouping scheme, the overall improvement is $142 \%$. When applying the 6-Frame and 12Frame grouping schemes, the overall improvement would rise to $147 \%$ and $149 \%$, respectively. However, the 6-Frame and 12-Frame grouping schemes further relax the control of guaranteeing each frame meeting its deadline.

\subsubsection{The Regulated Grouping Scheme}

From our simulation experiments, by the Regulated Schemes, we can improve the performance by $35-40 \%$. And by utilizing the Grouping Schemes, we can improve the performance by a dramatic $142 \%$. Table 7 shows the performance improvement for the Regulated Grouping Schemes as compare with the default transmission scheme.

\begin{tabular}{|l|c|c|c|c|}
\cline { 2 - 5 } \multicolumn{1}{c|}{} & \multicolumn{2}{c|}{ Local } & \multicolumn{2}{c|}{ Global } \\
\hline Type & Group3-Delay3 & Group6-Delay1 & Group3-Delay3 & Group6-Delay1 \\
\hline I & $117.98 \%$ & $128.03 \%$ & $121.16 \%$ & $121.61 \%$ \\
\hline II & $135.11 \%$ & $139.50 \%$ & $141.22 \%$ & $141.11 \%$ \\
\hline III & $166.16 \%$ & $170.30 \%$ & $167.07 \%$ & $167.52 \%$ \\
\hline IV & $184.84 \%$ & $208.64 \%$ & $210.62 \%$ & $212.66 \%$ \\
\hline Overall & $145.82 \%$ & $153.20 \%$ & $151.61 \%$ & $151.99 \%$ \\
\hline
\end{tabular}

Table 7. Performance improvement with the Regulated Grouping Schemes. 
From Table 7, the Regulated Grouping Scheme performs the best among the three proposed transmission schemes and the overall improvement is about $146 \%$. There is a clear trend that shows the Regulated Grouping Scheme works better on bursty traffic. The improvement for Type I to Type IV traffic under the grouping of 3 frames with a maximum delay of 3 frame-time are 118\%, 135\%, 166\%, and $185 \%$, respectively.

For the local Regulated Scheme, grouping 6 frames at a time is slightly better than grouping 3 frames at a time. However, when applying the Regulated Grouping Scheme globally, there is no different in performance between grouping 3 frames and grouping 6 frames together for transmission. With these experiment results, we therefore can utilize the combined transmission scheme to improve the transmission performance at the expenses of some start up delays and some frames occasionally missing their deadlines.

\section{Summary \& Future Work}

For a summary of this performance study, we first categorized the MPEG streams into 4 different types according to their I:P:B ratios. By examining the bandwidth demand and the transmission sequence, we proposed two ways to improve the support of a timed token MAC network for MPEG video transmission. With the Regulated Scheme, video streams are regulated such that they are not competing the bandwidth at the same time, thus, the unused bandwidth can be utilized. With this scheme, the percentage of improvement ranged from $21 \%$ to 59\% depending on the type of traffic. We also observed that by grouping subsequent frames together and forming a mega-frame, we could smooth out the fluctuation of bandwidth demand. With our simulation experiments, we found out that the 3-Frame grouping scheme can increase the performance by 113 to $191 \%$ depending on the type of traffic. We further our study in combining the Regulated Scheme with the Grouping Scheme. Our simulation experiments has shown that the Regulated Grouping Scheme indeed produced the best results. Hence, with an improvement ranging from $118 \%$ to $213 \%$, the combined scheme is a good compromise between transmission performance and video quality.

As for future work, we want to apply the imprecise computation model on MPEG video transmission such that we can transmit video with a guaranteed quality of service (QoS). Knowing that the I-frames are more important than the P- and B-frames, we will investigate transmission schemes that will occasionally skip the B-frames for improving performance in terms of synchronization but still guarantee that every I-frame and P-frame can meet their deadlines. We also plan to look into the problem of regulating the transmission rates at the sending stations so as to increase the support of a timed token MAC network in transmitting MPEG videos.

\section{References}

[AGR94] G. Agrawal, B. Chen, W. Zhao, and S. Davari, "Guaranteeing Synchronous Message Deadlines with the Timed Token Medium Access Control Protocol”, IEEE Transaction on Computers, vol. 43, No. 3, pp. 327 --- 339, March 1994.

[ANS85] IEEE/ANSI Standard 802.4 --- 1985 "Token passing bus access method and physical layer specifications", IEEE, New York, 1985.

[ANS87a] FDDI Token ring media access control (MAC). ANSI Standard X3.139, 1987.

[ANS87b] FDDI Token ring station management, draft proposal --- ANSI Standard X3T9.5 rev. 5, May 10, 1989.

[CHE92] B. Chen, G. Agrawal, and W. Zhao, "Optimal synchronous capacity allocation for hard real-time communication with the time token protocol". In Proceedings Real-time Systems Symposium, pages 198--207, December 1992.

[COH89] M. D. Cohn, "A network architecture for advanced aircraft", in Proceedings of the IEEE Conference on Local Computer Network, pp. 358 --- 364, Oct., 1989.

[GRE89] D. T. Green, and D. T. Marlow, "SAFENET --- A LAN for navy mission critical systems", in Proc. $14^{\text {th }}$ Conf. On Local Computer Network, pp. 340 --- 346, Oct., 1989. 
[GRO82] R. M. Grow, "A timed token protocol for local area networks", in Proc. Electro/82, Token Access Protocols, May 1982.

[HAN95] Han, Ching-Chih, and Kang G. Shih, "Scheduling MPEG-Compressed Video Streams with Firm Deadline Constraints," to be appeared in Proc. of the 3rd ACM International Multimedia Conference and Exhibition, November 1995.

[ISM95] M. R. Ismail, I. E. Lambadaris, M. Devetsikiotis, "Modeling Prioritized MPEG Video Using TES and Frame Spreading Strategy for Transmission in ATM Networks", Proc. IEEE INFOCOM, April 1995.

[IZQ95] R. Izquierdo and D. R. Reeves, "Statistical characterization of MPEG VBR video at the SLICE layer", Proc. SPIE Multimedia Computing and Networking, Vol. 2417, September 1995.

[KOC91] R. J. Kochanski, and J. L. Paige, "SAFENET --- The standard and its application", IEEE LCS, vol. 2, No. 1, pp. 46 --- 51, Feb., 1991.

[KRU95] M. Krunz, R. Sass, H. Hughes, "Statistical Characteristics and Multiplexing of MPEG Streams", Proc. IEEE INFOCOM, April 1995.

[LAM94] Lam, Simon S., Simon Chow, and David K.Y. Yau, "In Algorithm for Lossless Smoothing of MPEG Video," Proc. of ACM SIGCOMM 94, pp. 281-293, London England UK, 1994.

[MAL93] N. Malcolm and W. Zhao, "Guaranteeing synchronous messages with arbitrary deadline constraints in an fddi network". In Proceedings 18th Conference on Local Computer Networks, pages 186 -- 195. IEEE, 1993.

[MIN96] L. Ming, S. Koppenhoefer, "Real-Time Communication in a Simplified Timed Token Protocol", Proceedings of the International Conference on Multimedia Technology, Dec., 1996.

[NG91] J.K. Ng and J.W.S. Liu, "Performance of local area network protocols for hard real-time applications". In Proceedings 11 th International Conference of Distributed Computing Systems, pages 318--326. IEEE, May 1991.

[NG93A] J.K. Ng, "The support of different network medium-access protocols for multimedia applications". In Proceedings International Conference on Parallel and Distributed Systems, pages 343 -- 347, December 1993.

[NG93B] J.K. Ng, and J.W.S. Liu, "Performance of high-speed networks for multimedia applications". In Proceedings 18th Conference on Local Computer Networks, pages 513 -- 522. IEEE, September 1993.

[NG93C] J.K. Ng, Performance of High-Speed Networks for Real-time Applications, PhD thesis, University of Illinois at Urbana-Champaign, 1993.

[OTT92] Ott, Teunis, T. V. Lakshman, and Ali Tabatabai, "Scheme for Smoothing Delay-sensitive Traffic Offered to ATM Networks," Proc. of IEEE INFOCOM'92, pp. 776-785, 1992.

[PAI90] J. L. Paige, "SAFENET --- A navy approach to computer networking", in Proc. IEEE Conf. on Local Computer Network, pp. 268 --- 273, Oct., 1990.

[PAN92] Pancha, Pramond and Magda El Zarki, "A Look at the MPEG Video Coding Standard for Variable Bit Rate Video Transmission," Proc. of IEEE INFOCOM'92, pp. 85-94, 1992.

[PAN93] Pancha, Pramond and Magda El Zarki, "Bandwidth Requirements of Variable Bit Rate MPEG Sources in ATM Networks," Proc. of IEEE INFOCOM'93, pp. 902-909, 1993.

[REI92] Reibman, Amy R. and Arthur W. Berger, "On VBR Video Teleconferencing over ATM Networks," Proc. of IEEE GLOBECOM'92, pp. 314-319, 1992.

[REI93] Reininger, D. Raychaudhuri, B. Melamed, B. Sengupta, and J. Hill, "Statistical Multiplexing of VBR MPEG Compressed Video on ATM Networks," Proc. of IEEE INFOCOM'93, pp. 919-926, 1993.

[SAE88] Aerospace Systems Division, Committee AS-2, "Linear token-passing multiple data bus", AS4074.1 Version 4.0, Jan. 25, 1988.

[SEV87] K. C. Sevcik, and M. J. Johnson, "Cycle time properties of the FDDI token ring protocol" IEEE Transaction on Software Engineering, Vol. SE-13, No. 3, pp. 376 --- 385, 1987.

[SHA92] A. Shah, D. Staddon, I. Rubin, and A. Ratkovic, "Multimedia over FDDI", Proc. $17^{\text {th }}$ Conference on Local Computer Networks, pp. 110 --- 124, 1992.

[UHL91] R. W. Uhlhorn, "The fibre-optic high-speed data bus for a new generation of military aircraft", IEEE LCS, Vol. 2, No. 1, pp. 36 -- 45, Feb. 1991.

[ULM82] J. N. Ulm, "A timed token ring local area network and its performance characteristics", in Proc. Conference on Local Computer Network, pp. 50 --- 56, Feb. 1982.

[ZHA94] N. Malcolm, and W. Zhao, "The timed-token protocol for real-time communications". IEEE Computer, 27(1):35 -- 41. January 1994. 


\section{Appendix A}

The table below shows the number of MPEG video streams supported by different transmission schemes.

Regulated

Regulated Grouping

Video FDDI Local Global Grouping Local Global

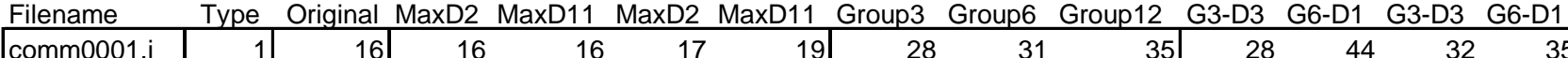

comm0002.i

comm0003.i

comm0010.i

comm0011.i

gpx0.i

racing01.i

racing02.i

racing03.i

racing04.i

racing05.i

basebal0.i

bdmin001.i

bdmin002.i

foot0001.i

foot0002.i

foot0003.i

foot0004.i

foot0005.i

tennis0.i

tennis01.i

tennis02.i

tennis03.i

tennis05.i

tennis1.i

tennis10.i

tennis11.i

tennis12.i

tennis13.i

tennis14.i

tennis2.i

anim0001.i

anim0002.i

anim0003.i

b_and_w.i

mtv00001.i

mtv00002.i

mtv00003.i

mtv00004.i

mtv00005.i

mtv00006.i

soap0.i

soap1.i

soapsub1.i

soapsub2.i

subsoap0.i

subsoap1.i

news0001.i

news0002.i

news0003.i

still001.i

telemkt0.i

\begin{tabular}{|c|c|c|c|c|c|c|c|c|c|c|c|}
\hline 16 & 16 & 16 & 17 & 19 & 28 & 31 & 35 & 28 & 44 & 32 & 35 \\
\hline 36 & 85 & 57 & 53 & 56 & 88 & 87 & 86 & 87 & 87 & 88 & 87 \\
\hline 30 & 30 & 30 & 54 & 41 & 84 & 88 & 88 & 90 & 89 & 90 & 89 \\
\hline 36 & 56 & 56 & 85 & 63 & 87 & 86 & 85 & 86 & 86 & 86 & 86 \\
\hline 31 & 52 & 52 & 50 & 42 & 61 & 61 & 61 & 62 & 62 & 62 & 61 \\
\hline 28 & 29 & 29 & 36 & 37 & 50 & 49 & 49 & 49 & 49 & 50 & 49 \\
\hline 19 & 19 & 19 & 29 & 29 & 46 & 50 & 52 & 52 & 56 & 53 & 53 \\
\hline 43 & 30 & 30 & 41 & 39 & 59 & 58 & 58 & 58 & 58 & 59 & 60 \\
\hline 31 & 68 & 49 & 38 & 57 & 76 & 76 & 76 & 76 & 76 & 77 & 76 \\
\hline 45 & 58 & 58 & 43 & 39 & 80 & 80 & 80 & 80 & 80 & 80 & 80 \\
\hline 28 & 30 & 30 & 53 & 38 & 63 & 63 & 65 & 66 & 64 & 64 & 64 \\
\hline 30 & 30 & 30 & 53 & 56 & 109 & 108 & 107 & 106 & 109 & 109 & 108 \\
\hline 26 & 29 & 29 & 32 & 29 & 60 & 59 & 59 & 60 & 59 & 60 & 60 \\
\hline 23 & 23 & 23 & 25 & 29 & 49 & 50 & 53 & 50 & 51 & 53 & 53 \\
\hline 26 & 25 & 25 & 30 & 32 & 61 & 64 & 64 & 64 & 64 & 64 & 64 \\
\hline 26 & 26 & 26 & 37 & 34 & 54 & 53 & 53 & 53 & 53 & 54 & 53 \\
\hline 30 & 49 & 49 & 37 & 46 & 68 & 68 & 68 & 68 & 68 & 68 & 68 \\
\hline 38 & 70 & 70 & 68 & 57 & 79 & 79 & 78 & 78 & 78 & 79 & 79 \\
\hline 18 & 18 & 18 & 20 & 22 & 40 & 42 & 43 & 43 & 44 & 43 & 44 \\
\hline 30 & 27 & 27 & 39 & 47 & 76 & 83 & 84 & 85 & 84 & 85 & 84 \\
\hline 30 & 30 & 30 & 32 & 34 & 74 & 73 & 73 & 74 & 73 & 74 & 73 \\
\hline 28 & 29 & 29 & 36 & 40 & 69 & 69 & 68 & 69 & 69 & 69 & 69 \\
\hline 64 & 80 & 80 & 82 & 58 & 89 & 89 & 88 & 89 & 89 & 89 & 89 \\
\hline 28 & 29 & 29 & 42 & 37 & 84 & 84 & 83 & 82 & 84 & 84 & 84 \\
\hline 27 & 48 & 48 & 44 & 33 & 81 & 81 & 80 & 81 & 81 & 81 & 81 \\
\hline 51 & 62 & 62 & 62 & 68 & 128 & 128 & 127 & 128 & 128 & 128 & 128 \\
\hline 41 & 62 & 62 & 62 & 64 & 88 & 88 & 88 & 88 & 88 & 89 & 88 \\
\hline 68 & 67 & 82 & 82 & 73 & 82 & 82 & 81 & 82 & 82 & 82 & 82 \\
\hline 20 & 20 & 20 & 25 & 25 & 43 & 45 & 46 & 37 & 45 & 46 & 47 \\
\hline 29 & 49 & 49 & 36 & 41 & 78 & 75 & 78 & 77 & 76 & 78 & 79 \\
\hline 46 & 59 & 62 & 59 & 62 & 120 & 124 & 123 & 108 & 124 & 124 & 124 \\
\hline 30 & 26 & 26 & 36 & 56 & 89 & 94 & 96 & 109 & 108 & 99 & 99 \\
\hline 36 & 48 & 48 & 41 & 42 & 86 & 84 & 86 & 83 & 83 & 86 & 86 \\
\hline 26 & 27 & 27 & 35 & 34 & 63 & 69 & 70 & 76 & 83 & 70 & 72 \\
\hline 20 & 20 & 20 & 29 & 29 & 52 & 56 & 58 & 59 & 59 & 58 & 58 \\
\hline 30 & 29 & 29 & 50 & 45 & 97 & 96 & 98 & 97 & 96 & 98 & 100 \\
\hline 35 & 98 & 66 & 66 & 54 & 110 & 110 & 109 & 104 & 110 & 111 & 110 \\
\hline 53 & 94 & 68 & 70 & 60 & 100 & 99 & 98 & 97 & 97 & 100 & 98 \\
\hline 34 & 91 & 91 & 64 & 79 & 109 & 108 & 107 & 108 & 108 & 109 & 108 \\
\hline 21 & 21 & 21 & 27 & 28 & 53 & 56 & 57 & 53 & 59 & 59 & 59 \\
\hline 48 & 89 & 65 & 83 & 33 & 99 & 99 & 99 & 99 & 99 & 99 & 99 \\
\hline 64 & 142 & 92 & 92 & 86 & 172 & 176 & 175 & 178 & 178 & 178 & 177 \\
\hline 74 & 82 & 115 & 78 & 63 & 145 & 144 & 144 & 143 & 142 & 144 & 144 \\
\hline 26 & 25 & 25 & 37 & 35 & 69 & 70 & 72 & 71 & 70 & 71 & 72 \\
\hline 26 & 23 & 23 & 35 & 42 & 64 & 69 & 70 & 75 & 75 & 73 & 73 \\
\hline 34 & 51 & 51 & 61 & 61 & 90 & 90 & 90 & 90 & 90 & 90 & 90 \\
\hline 37 & 45 & 45 & 36 & 44 & 73 & 75 & 77 & 74 & 76 & 79 & 79 \\
\hline 36 & 78 & 80 & 78 & 63 & 127 & 131 & 135 & 125 & 137 & 138 & 138 \\
\hline 32 & 58 & 58 & 57 & 50 & 113 & 118 & 122 & 111 & 123 & 123 & 124 \\
\hline 42 & 61 & 61 & 61 & 63 & 114 & 118 & 122 & 115 & 120 & 123 & 124 \\
\hline 43 & 53 & 53 & 39 & 39 & 93 & 100 & 101 & 93 & 100 & 100 & 102 \\
\hline 50 & 64 & 65 & 64 & 71 & 130 & 130 & 129 & 120 & 130 & 130 & 130 \\
\hline
\end{tabular}

